\title{
Contrasting Effects on Discrimination Learning after Hippocampal Lesions and Conjoint Hippocampal-Caudate Lesions in Monkeys
}

\author{
Edmond Teng, ${ }^{3}$ Lisa Stefanacci, ${ }^{2}$ Larry R. Squire, ${ }^{1,2,3,4}$ and Stuart M. Zola ${ }^{1,2,3}$ \\ ${ }^{1}$ Veterans Affairs Medical Center, San Diego, California 92161, and Departments of ${ }^{2}$ Psychiatry, ${ }^{3}$ Neurosciences, and \\ 4 Psychology, University of California, La Jolla, California 92093
}

\begin{abstract}
Eighteen monkeys with lesions of the hippocampal region (the hippocampus proper, the dentate gyrus, and the subiculum) made by an ischemic procedure, radio frequency, or ibotenic acid were tested on a simple, two-choice object discrimination learning task that has been shown to be sensitive to large lesions of the medial temporal lobe. The monkeys were also tested on two other discrimination tasks (pattern discrimination and eight-pair concurrent discrimination) that can be learned normally by monkeys with large medial temporal lobe lesions. All of the lesion groups were impaired at learning the simple object discrimination task. Seven of the monkeys who had sustained damage to the hippocampal region also sustained damage to the tail of the caudate nucleus. These seven mon-
\end{abstract}

keys, but not the other 11 monkeys with hippocampal lesions, were impaired on pattern discrimination and concurrent discrimination learning. The results suggest that the hippocampal region is important for learning easy, two-choice discriminations, whereas the caudate nucleus is necessary for the normal learning of more difficult, gradually acquired discrimination tasks. The findings support the distinction between declarative memory, which depends on the hippocampus and related medial temporal lobe structures, and habit learning, which depends on the caudate nucleus.

Key words: hippocampus; caudate nucleus; monkeys; discrimination learning; radio frequency lesions; ischemic lesions; ibotenic acid lesions
In the years after the identification of the components of the medial temporal lobe memory system in the monkey (Squire and Zola-Morgan, 1991), efforts have been directed toward understanding how selective damage to medial temporal lobe structures affect memory (Mishkin and Murray, 1994; Mishkin et al., 1999; Zola and Squire, 2000). The hippocampus has been a particular focus of interest because, in humans, damage limited to the hippocampus itself is sufficient to cause clinically significant memory impairment (Zola-Morgan et al., 1986; Rempel-Clower et al., 1996).

Patients with hippocampal damage are forgetful and are deficient at any number of memory tasks that assess recognition, recall, or cued recall of recently encountered material. Accordingly, one might expect that a wide variety of memory tasks should be sensitive to the effects of hippocampal lesions in the monkey. However, this simple idea is complicated by the fact that some tasks that are failed by amnesic patients can be accomplished quite well by monkeys with the same or similar lesions. For example, amnesic patients are impaired at the learning and retention of concurrent discrimination tasks (Squire et al., 1988), whereas even monkeys with large medial temporal lobe lesions learn this task normally (Buffalo et al., 1998).

One of the tasks that has been the topic of considerable work and discussion is the object discrimination task. In this task,

Received Nov. 29, 1999; revised Feb. 22, 2000; accepted Feb. 22, 2000.

This research was supported by the Medical Research Service of the Department of Veterans Affairs, National Institutes of Health Grants MH58933, MH24600, MH11649, MH18399, and MH11154, and the McDonnell-Pew Center for Cognitive Neuroscience. We thank Cecelia Manzanares, Elizabeth Buffalo, Jeff Manzanares, Scott Hanson, Michelle $\mathrm{Hu}$, Elaine Ellerton, and Jennifer Frascino for technical assistance.

Correspondence should be addressed to Dr. Stuart M. Zola, Department of Psychiatry, University of California, San Diego School of Medicine, La Jolla, CA 92093. E-mail: szola@ucsd.edu.

Copyright (C) 2000 Society for Neuroscience $\quad 0270-6474 / 00 / 203853-11 \$ 15.00 / 0$ monkeys learn over several trials which object (of two that are presented together) is always rewarded. Typically, the objects are easily discriminable, and normal monkeys can learn the task in a single test session. Object discrimination tasks are best viewed as a subset of visual discrimination problems involving only one pair of stimuli. The stimuli in visual discrimination tasks can be two different colors, different brightnesses, they can be plaques displaying two-dimensional patterns (e.g., $\mathrm{N}$ vs W), they can be two images on a computer monitor, or they can be objects.

Amnesic patients are impaired at learning simple object discrimination problems and at retaining them across intervals of 1-11 d (Squire et al., 1988). However, the results with monkeys that have accumulated over the years have been inconsistent. Several studies have reported impaired learning of object discriminations after medial temporal lobe lesions or more restricted hippocampal lesions $\left(\mathrm{H}^{+} \mathrm{A}^{+}\right.$group, Zola-Morgan and Squire, 1985; $\mathrm{H}^{+}$group, Zola-Morgan et al., 1989a; $\mathrm{H}^{+}$A group, ZolaMorgan et al., 1989b; PRPH group, Zola-Morgan et al., 1989c; ISC group, Zola-Morgan et al., 1992; $\mathrm{H}^{++}$group, Zola-Morgan et al., 1993). Yet, other studies have found the initial learning of object discriminations to be unimpaired (Orbach et al., 1960; Jones and Mishkin, 1972; Mahut et al., 1981) (PRPH II group, Suzuki et al., 1993; H group, Alvarez et al., 1995; Doré et al., 1998).

One source of confusion has been that object discrimination tasks have sometimes been discussed together with other kinds of discrimination learning tasks (e.g., concurrent discrimination learning and pattern discrimination learning), when in fact the tasks are different in important ways. In the object discrimination task, one pair of objects is trained at a time, and learning occurs quickly within a single session. Concurrent discrimination learning (which requires that an animal learn several pairs of objects concurrently) and pattern discrimination learning are learned 
gradually over hundreds of trials. Iversen (1976) suggested that monkeys might learn these more difficult discrimination tasks in the way that humans learn motor skills. Subsequently, it was suggested that slowly acquired discrimination tasks may depend on a cortico-striatal, noncognitive, habit learning system, which functions independently of the medial temporal lobe memory system (Mishkin et al., 1984). It has been unclear how simple object discrimination learning should be viewed in the light of this distinction and, specifically, whether object discrimination learning does or does not depend on the integrity of the hippocampus or other medial temporal lobe structures.

We have evaluated the performance of 18 monkeys with lesions of the hippocampal region on three discrimination tasks: object discrimination learning, concurrent discrimination learning, and pattern discrimination learning. Seven of the monkeys sustained damage to the tail of the caudate nucleus in addition to hippocampal damage. We asked whether object discrimination learning is affected by hippocampal damage and how caudate nucleus damage affects performance on each task.

\section{MATERIALS AND METHODS}

\section{Subjects}

Behavioral findings from 34 male cynomolgus monkeys (Macaca fascicularis) are presented (Table 1). The animals weighed between 3.9 and 5.8 $\mathrm{kg}$ at the start of testing. Four groups had intended lesions of the hippocampal region (the hippocampus proper, the dentate gyrus, and subiculum). One of the groups had ischemic lesions (group ISC; $n=4$ ), two groups had radio frequency lesions of the hippocampal region (group $\mathrm{RF} 1, n=4$ and group RF2, $n=5$ ), and the fourth group had bilateral ibotenate lesions of the hippocampal region (group IBO1, $n=5$ ). The lesions in the IBO1 group were intended to damage the cell bodies of the hippocampal region while sparing white matter and adjacent medial temporal lobe structures (the amygdala and entorhinal, perirhinal, and parahippocampal cortices). For two of the operated groups, some of the data presented here have been presented previously (group ISC, ZolaMorgan et al., 1992; group RF1 in Alvarez et al., 1995). Additionally, sixteen unoperated normal monkeys (group N) served as controls. They had all completed testing on our standard memory battery, including the three tasks described here. Data from 10 of these monkeys have been published previously (N1-N7, Alvarez et al., 1995; N8-N10, Buffalo et al., 1998).

\section{Surgery, histological processing, and histological analysis}

Detailed descriptions of the procedures used in the surgical preparation, histological processing, and histological analysis for all of the monkeys with hippocampal lesions have been published previously (group ISC, Zola-Morgan et al., 1992; the RF1 group in Alvarez et al., 1995; the RF2 and IBO1 groups, Zola et al., 2000).

Surgical preparation. For the ISC group, each monkey was subjected to $15 \mathrm{~min}$ of reversible ischemia, using a noninvasive technique that combined carotid occlusion and pharmacologically induced hypotension. For the RF1 and RF2 groups, magnetic resonance (MR) images of each monkey's brain were obtained by placing each monkey in a custom-built nonmetallic acrylic stereotaxic head holder. Radio-opaque beads, anchored to the monkey's skull with dental acrylic, served as landmarks on the MR images from which stereotaxic coordinates for the intended lesions could be derived. Using a specially designed electrode connected to a radio frequency lesion maker, seven overlapping lesions were produced along the rostrocaudal extent of the hippocampal region on each side of the brain. For the IBO1 group, imaging and surgical procedures similar to those described for the RF groups were used. A Hamilton syringe, filled with ibotenic acid $(10 \mathrm{mg} / \mathrm{ml}$ in a $0.1 \mathrm{M}$ phosphate buffer solution), was used to produce seven overlapping lesions along the rostrocaudal extent of the hippocampus bilaterally. At each lesion site, $0.8 \mu \mathrm{l}$ of ibotenic acid was slowly injected during $5 \mathrm{~min}$.

Histological procedures. Monkeys were administered an overdose of Nembutal and perfused transcardially with $200 \mathrm{ml}$ of a buffered $0.9 \%$ $\mathrm{NaCl}$ solution, followed by 21 of $10 \%$ formaldehyde solution (in $0.1 \mathrm{M}$ phosphate buffer) at a rate of $100 \mathrm{ml} / \mathrm{min}$. Brains were then blocked in situ in the coronal plane, removed from the skull, cryoprotected first in a $10 \%$ glycerol-10\% formaldehyde solution (in $0.1 \mathrm{M}$ phosphate buffer) and then in a $20 \%$ glycerol-10\% formaldehyde solution, and subsequently quick-frozen in isopentane at $-78^{\circ} \mathrm{C}$. Using a freezing microtome, coronal sections were cut at a thickness of $50 \mu \mathrm{m}$ beginning just anterior to the hippocampus and continuing caudally through the length of the hippocampal region (for the ISC group, sections were cut at a thickness of $30 \mu \mathrm{m}$ ). One series (every fifth section) was mounted and stained with thionin to assess the extent of the lesions (for the ISC group, every eighth section was mounted and stained).

Histological analyses. Detailed histological analyses were performed for the hippocampal region, as well as for two other brain areas (the parahippocampal cortex and the caudate nucleus) that were found to have sustained more than minimal damage.

Damage to the hippocampal region. For each monkey in the ISC group, thionin-stained sections were examined at $0.96 \mathrm{~mm}$ intervals (every fourth stained section) along the rostrocaudal extent of the hippocampal region. Camera lucida drawings of the perimeter of the CA1 field were then made from each slide at a $30 \times$ magnification and traced using a digitizing tablet to compute an areal measurement for each section. For each brain, the measurements for each level were added together, and the sum was multiplied by the interslice interval $(0.96 \mathrm{~mm})$ to obtain an estimate of the spared CA1 volume. The overall measurement of CA1 volume in the ISC group was compared with measurements of CA1 volume obtained from four, weight-matched, unoperated control monkeys.

For each monkey in the RF1, RF2, and IBO1 groups, thionin-stained sections were examined at $0.5 \mathrm{~mm}$ intervals along the rostrocaudal extent of the hippocampal region. Each section was scanned into a Power Macintosh G3 computer (Apple Computers, Cupertino, CA) using a ScanMaker 4 scanner. The structures comprising the hippocampal region (the dentate gyrus, the cell fields of the hippocampus proper, and subiculum) were classified on the basis of cytoarchitectonics using a light microscope (WILD 3Z; Leica, Nussloch, Germany), and the boundaries for the hippocampal region were marked on the computerized images of each section. Using NIH Image and Canvas, bilateral measures of the cross-sectional area of the hippocampal region were obtained from each section. For each brain, the cross-sectional area for each section was multiplied by the interslice interval, and the estimates from each section were added together to obtain a measure of the volume of the spared hippocampal region. Then, the overall measure of spared hippocampal region volume for each monkey with IBO or RF lesions was subtracted from the average measures of hippocampal region volume from three weight-matched, unoperated control monkeys to obtain a measure of percent damage. The percent damage to the regions that included the CA1/subiculum, the CA3/dentate gyrus, the anterior half of the hippocampal region, and the posterior half of the hippocampal region, were also determined using the procedures just described.

Damage to the parahippocampal cortex and the caudate nucleus. For each monkey, brain sections were examined at $1 \mathrm{~mm}$ intervals along the rostrocaudal extent of the temporal lobe (range of 16-19 sections), and the same procedures used to determine the extent of damage to the hippocampal region were used to determine the percent damage to the parahippocampal cortex. These same procedures were also used to determine the extent of damage to the tail of the caudate nucleus (from level A 12.1 to level A 4.6; Szabo and Cowan, 1984).

\section{Behavioral testing}

Before the behavioral testing described in this report, all five groups of monkeys had been tested on the trial-unique delayed nonmatching to sample task. Additionally, the IBO1 group had received preoperative and postoperative testing on the visual paired-comparison task before testing on the trial-unique delayed nonmatching to sample task (Zola et al., 2000). Monkeys were then tested on the following three tasks in the order described.

\section{Pattern discrimination}

Monkeys were tested on two separate two-choice pattern discrimination tasks for which cues of color, size, and three-dimensional shape were not available. In the first task, the animals learned to discriminate a plus sign from a square, and in the second task to discriminate an $\mathrm{N}$ from a $\mathrm{W}$. The stimuli were constructed from strips of white tape placed on identical gray plaques. For each trial, the plaques were placed over the two lateral food wells in the Wisconsin General Testing Apparatus (WGTA). A correct choice uncovered a raisin reward, and an incorrect choice uncovered an empty food well. The position of the correct plaque (over the left 


\begin{tabular}{|c|c|c|c|c|c|c|c|}
\hline Monkey & $\begin{array}{l}\text { Hippocampal } \\
\text { region }\end{array}$ & $\begin{array}{l}\text { Anterior } \\
\text { HR }\end{array}$ & $\begin{array}{l}\text { Posterior } \\
\text { HR }\end{array}$ & $\begin{array}{l}\text { CA3 and } \\
\text { dentate }\end{array}$ & $\begin{array}{l}\text { CA1 and } \\
\text { subiculum }\end{array}$ & $\begin{array}{l}\text { PH } \\
\text { cortex }\end{array}$ & $\begin{array}{l}\text { Caudate } \\
\text { nucleus }\end{array}$ \\
\hline ISC1 & NA & NA & NA & NA & 24 & 0 & 0 \\
\hline ISC2 & NA & NA & NA & NA & 73 & 0 & 0 \\
\hline ISC3 & NA & NA & NA & NA & 24 & 0 & 0 \\
\hline ISC4 & NA & NA & NA & NA & 24 & 0 & 0 \\
\hline Mean & & & & & 36 & 0 & 0 \\
\hline RF1-1 & 60 & 61 & 58 & 52 & 65 & 0 & 19 \\
\hline RF1-2 & 63 & 61 & 68 & 69 & 57 & 0 & 37 \\
\hline RF1-3 & 47 & 44 & 53 & 36 & 57 & 38 & 0 \\
\hline RF1-4 & 76 & 77 & 72 & 75 & 77 & 0 & 0 \\
\hline Mean & 62 & 61 & 63 & 58 & 64 & 10 & 14 \\
\hline RF2-1 & 15 & 20 & 5 & 22 & 6 & 0 & 72 \\
\hline RF2-2 & 35 & 40 & 25 & 53 & 14 & 0 & 57 \\
\hline RF2-3 & 23 & 28 & 12 & 40 & 2 & 0 & 70 \\
\hline RF2-4 & 10 & 14 & 4 & 5 & 15 & 0 & 85 \\
\hline RF2-5 & 39 & 47 & 22 & 55 & 21 & 0 & 79 \\
\hline Mean & 24 & 30 & 14 & 35 & 12 & 0 & 73 \\
\hline IBO1-1 & 40 & 15 & 88 & 25 & 57 & 10 & 0 \\
\hline IBO1-2 & 52 & 42 & 73 & 46 & 61 & 0 & 0 \\
\hline IBO1-3 & 53 & 42 & 73 & 54 & 49 & 7 & 0 \\
\hline IBO1-4 & 39 & 28 & 60 & 17 & 64 & 46 & 0 \\
\hline IBO1-5 & 34 & 38 & 25 & 20 & 48 & 23 & 0 \\
\hline Mean & 44 & 33 & 64 & 32 & 56 & 17 & 0 \\
\hline
\end{tabular}

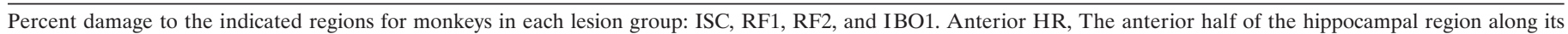

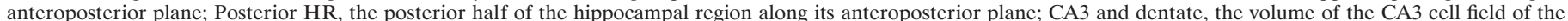

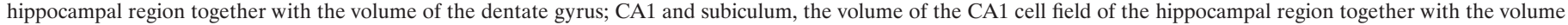

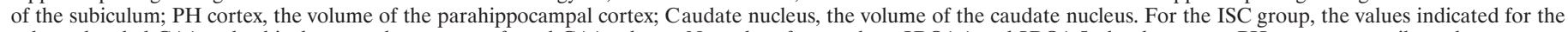
column headed CA1 and subiculum are the percent of total CA1 volume. Note that, for monkeys IBO1-1 and IBO1-5, the damage to PH cortex was unilateral.

or right lateral well) varied on each trial according to a pseudorandom schedule (Gellerman, 1933). Monkeys received 30 trials per day, and testing continued until a learning criterion of 27 correct responses in 30 trials $(90 \%)$ was achieved on 2 consecutive days.

\section{Simple object discrimination learning}

Monkeys were tested on four separate two-choice object discrimination tasks (referred to previously as delayed retention of object discriminations, e.g., Alvarez et al., 1995). Each discrimination problem involved a pair of distinctive objects that could be learned by normal monkeys within a single session. The four discriminations consisted of a red versus a green peanut shell, a white versus a black rectangle, a pink versus a yellow plastic eggshell, and a piece of a real Oreo cookie versus a piece of plastic cookie. Each task was administered for two daily sessions of 20 trials each, with an intertrial interval of $15 \mathrm{sec}$. After a 2 d delay, an additional 20 trials were administered. The position of the correct object (over the left or right lateral well of the WGTA) varied on each trial according to a pseudorandom schedule (Gellerman, 1933). A raisin reward was pressed to the underside of the correct object and was revealed when that object was chosen. An interval of $3 \mathrm{~d}$ separated the administration of each discrimination problem. Monkeys N1, N6, N12, N13, ISC-3, ISC-4, RF1-1, RF1-3, and RF2-4 each exhibited motivational problems during testing on one of the four object discrimination pairs. The problematic object pair varied from monkey to monkey. For these monkeys, the data analysis was based on only three discrimination problems.

\section{Concurrent discrimination}

Monkeys were required to learn simultaneously eight pairs of objects. The objects varied in color, shape, and size, and were arranged in pairs that shared several features (e.g., several pairs included a blue object) (see also Buffalo et al., 1999 and the cover illustration from that issue of the journal). The pairs in each training session were presented randomly so that each pair was presented five times during the course of a single daily testing session of 40 trials. During each trial, one pair of objects was presented over the two lateral food wells of the WGTA. The same object in each pair was always correct. A correct choice uncovered a food reward, and an incorrect choice uncovered an empty food well. The position of the correct object (over the left or right lateral well) varied on each trial according to a pseudorandom schedule (Gellerman, 1933). Testing continued until a learning criterion of 39 correct responses in 40 consecutive trials was achieved within a single testing session.

\section{RESULTS}

\section{Neurohistological findings}

\section{The ISC and RF1 groups}

Neurohistological findings from the four monkeys in the ISC group and the four monkeys in the RF1 group have been published previously (ISC, Zola-Morgan et al., 1992; group RF1, Alvarez et al., 1995). Briefly, the four monkeys with ISC lesions sustained significant loss of pyramidal cells in the CA1 and CA2 fields of the hippocampus, as well as loss of somatostatinimmunoreactive cells in the hilar region of the dentate gyrus. Cell loss occurred bilaterally throughout the rostrocaudal extent of the hippocampus but was greater in the caudal portion. The damage within the CA1 pyramidal cell field averaged $24 \%$ of total CA1 volume in three of the monkeys and $73 \%$ in the monkey with the largest lesion (ISC2; Table 1). Animal ISC2 also sustained some subicular damage. Except for patchy loss of cerebellar Purkinje cells, significant damage was not detected in areas outside the hippocampus, including the adjacent entorhinal, perirhinal, and parahippocampal cortices. For the four monkeys in the RF1 
group, the mean percentage damage to the hippocampal region was $62 \%$ (range, $47-76 \%$ ). To maintain consistency in the percent damage measures across operated groups, the brains of the RF1 group were remeasured relative to the three control brains that were used to obtain the percent damage measures for the IBO1 and RF2 groups. Differences between the percent damage values reported in Alvarez et al. (1995) and the present study averaged $4.5 \%$. The perirhinal cortex was spared in all of the monkeys. There was damage to the entorhinal cortex in one monkey (RF1-3, 10\% overall damage), and this monkey also sustained slight to moderate damage to the parahippocampal cortex $(38 \%)$. Slight to moderate asymmetrical damage to white matter subjacent to the hippocampal region occurred in three animals (RF1-1, RF1-2, and RF1-3), and unilateral damage to the tail of the caudate nucleus occurred in two animals (RF1-1 and RF1-2). Figure 1 shows representative photomicrographs from monkeys in the ISC and RF1 groups.

\section{RF2 group}

Neurohistological findings for these five monkeys was presented by Zola et al. (2000). The mean percentage damage was $24 \%$ (range, 10\%-39\%) (Table 1). In all five animals, the damage to the hippocampal region was limited mainly to field CA3 and to the dentate gyrus. The lesions were intended to spare the most anterior portion of the hippocampal region to prevent inadvertent damage to the amygdala. However, in three of the animals (RF2-1, RF2-2, and RF2-4), the damage began more anterior than intended. Two of these animals (RF2-1 and RF2-2) had slight bilateral damage to the posterior portion of the amygdala, and one animal (RF2-4) had moderate bilateral damage to the posterior amygdala. The entorhinal, perirhinal, and parahippocampal cortices were spared in all five animals. There was bilateral damage to the tail of the caudate nucleus in all five animals (mean percent damage, $73 \%$ ), and all five animals sustained slight to moderate bilateral damage to the lateral aspect of the lateral geniculate nucleus (LGN) (range, 19\%-45\%). There was also slight to moderate bilateral damage to the anterior portion of the stria terminalis in all five monkeys. Figure 1 shows photomicrographs from a representative monkey in the RF2 group.

\section{IBO1 group}

Neurohistological findings for these five monkeys was presented by Zola et al. (2000). Overall, the monkeys in the IBO1 group sustained substantial bilateral damage to the hippocampal region (i.e., the cell fields of the hippocampus proper, the dentate gyrus, and the subiculum). The mean percentage damage for all five animals was $44 \%$ (range, 34-53\%) (Table 1). The most anterior portion of the hippocampal region was intentionally spared to prevent inadvertent damage to the amygdala, and the amygdala was entirely spared in all five animals. The entorhinal and perirhinal cortices sustained slight unilateral damage in one animal (IBO1-5), but were spared in the other four animals. The parahippocampal cortex was completely spared in monkey IBO1-2. There was slight to moderate unilateral damage to the posterior parahippocampal cortex in two animals (amounting to $\sim 10 \%$ on the left side in IBO1-1 and 23\% on the right side in IBO1-5), and slight to moderate bilateral damage to the parahippocampal cortex in two animals (amounting to $\sim 7 \%$ in monkey IBO1-3 and $46 \%$ in monkey IBO1-4). There was no damage to the tail of the caudate nucleus, the stria terminalis, or the lateral geniculate nucleus in any of the animals. Figure 1 shows photomicrographs from a representative monkey in the IBO1 group.

\section{Behavioral findings}

The behavioral results for the object discrimination task will be presented first, followed by the results from the pattern discrimination task and from the concurrent discrimination task.

\section{Simple object discrimination learning}

Table 2 shows the performance of the N, ISC, RF1, RF2, and IBO1 groups on the simple object discrimination task. Except as indicated in Materials and Methods, performance scores were averaged across all four object discrimination problems for each monkey. Two separate analyses were performed. We first examined performance across all 20 trials of each test day, an analysis used in previous reports that described the effects of hippocampal damage on this task (Zola-Morgan et al., 1989; Alvarez et al., 1995). In the second analysis, we examined performance on the first four trials of each testing day because studies of simple discrimination learning in human amnesic patients (Squire et al., 1988) had suggested that the first several trials of each testing day are the most sensitive to memory impairment (Fig. 2C) (see Discussion).

\section{Performance on all 20 trials of each test day}

Table 2 shows the performance of the 16 normal monkeys and the 18 monkeys with hippocampal lesions across the 20 trials of each test day. A two-way repeated-measures ANOVA (16 normal monkeys vs 18 operated monkeys across days 1,2 , and 4) indicated a marginally significant effect of group $\left(F_{(1,32)}=3.6 ; p=\right.$ $0.066)$, a significant effect of day $\left(F_{(2,64)}=157.2 ; p<0.0001\right)$, and no group $\times$ day interaction $\left(F_{(2,64)}=1.0 ; p>0.10\right)$. Separate comparisons of each lesion group with the $\mathrm{N}$ group indicated that the ISC group was impaired on day 1 (ISC, 68\% correct; N, 77\% correct; $p<0.05$ ) and that the RF2 group was marginally impaired on day 2 (RF2, 86\% correct; N, 91\% correct; $p=0.058$ ). Thus, when the data for all 20 trials were considered, there was limited evidence for an impairment in the monkeys with hippocampal lesions.

\section{Performance on the first four trials of each test day}

Figure $2 A$ shows the learning curves (in blocks of four trials) for the two groups across the 3 test days. Table 2 shows the performance of the 16 normal monkeys and the 18 monkeys with hippocampal lesions on the first four trials of each test day. A two-way repeated-measures ANOVA (normal vs operated monkeys for the first four trials of days 1,2, and 4) indicated an effect of group $\left(F_{(1,32)}=8.1 ; p<0.01\right)$, an effect of day $\left(F_{(2,64)}=126.4\right.$; $p<0.0001)$, and a group $\times$ day interaction $\left(F_{(2,64)}=7.0 ; p<\right.$ $0.01)$.

Figure 2, $B$ and $C$, shows the performance of the monkeys compared with the performance of nine amnesic patients who had been given the same task in a previous study (Squire et al., 1988). The 18 monkeys with lesions of the hippocampal region $(\mathrm{H})$ were impaired on the first four trials of day $1(\mathrm{H}, 56 \%$ correct; $\mathrm{N}, 71 \%$ correct; $p<0.01)$ and on the first four trials of day $2(\mathrm{H}, 82 \%$ correct; N, 88\% correct; $p<0.05)$ but not impaired on day $4(\mathrm{H}$, $95 \%$ correct; N, 95\% correct; $p>0.10)$. The amnesic patients were also impaired on the first four trials of days 1 and $2(p<$ $0.05)$. Finally, when performance of the monkeys and the patients was evaluated across either the first three trials or the first five trials, the results were similar to the findings for the first four trials (for days 1 and 2 , all $p$ values $<0.05$ ). Thus, there was clear 

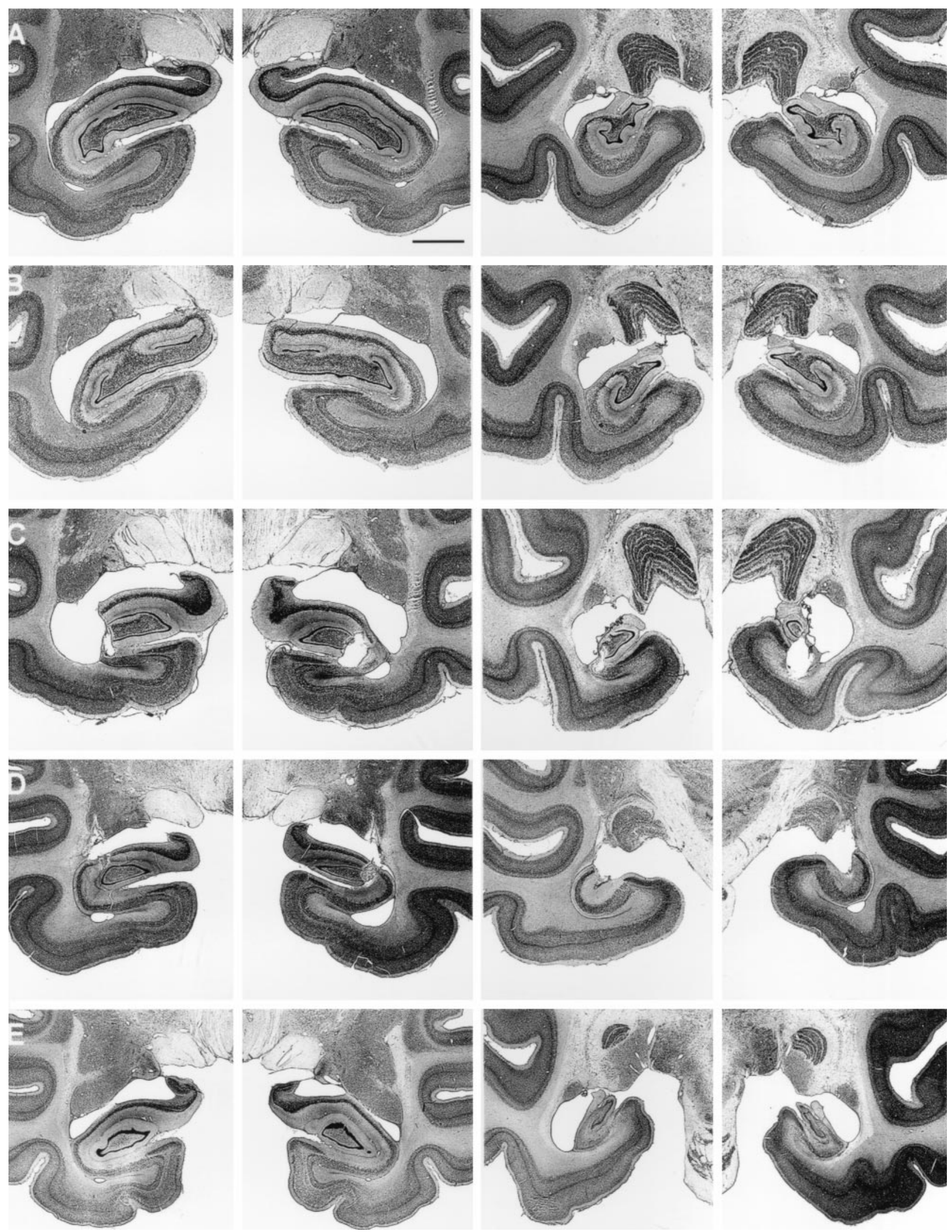

Figure 1. Photomicrographs of thionin-stained sections through two levels of the left and right temporal lobe of a normal monkey $(A)$ and a representative monkey from the ISC $(B), \mathrm{RF} 1(C), \mathrm{RF} 2(D)$, and IBO1 $(E)$ groups. Scale bar (in $A): A-E, 2 \mathrm{~mm}$. 
Table 2. Visual discrimination learning

Simple object discrimination learning

\begin{tabular}{|c|c|c|c|c|c|c|c|c|}
\hline \multirow[b]{2}{*}{ Group } & \multicolumn{3}{|c|}{ All 20 trials } & \multicolumn{3}{|c|}{ First four trials } & \multirow{2}{*}{$\begin{array}{l}\text { Pattern } \\
\text { discrimination }\end{array}$} & \multirow{2}{*}{$\begin{array}{l}\text { Concurrent } \\
\text { discrimination }\end{array}$} \\
\hline & Day 1 & Day 2 & Day 4 & Day 1 & Day 2 & Day 4 & & \\
\hline \multicolumn{9}{|l|}{$\mathrm{N}$} \\
\hline 1 & 77 & 95 & 93 & 67 & 83 & 100 & 480 & 240 \\
\hline 2 & 75 & 93 & 95 & 44 & 94 & 100 & 360 & 720 \\
\hline 3 & 71 & 90 & 93 & 69 & 75 & 94 & 240 & 640 \\
\hline 4 & 75 & 90 & 92 & 42 & 75 & 83 & 390 & 240 \\
\hline 5 & 75 & 86 & 93 & 56 & 69 & 81 & 1000 & 360 \\
\hline 6 & 77 & 88 & 93 & 66 & 83 & 92 & 450 & 480 \\
\hline 7 & 76 & 90 & 95 & 81 & 94 & 94 & 860 & 600 \\
\hline 8 & 70 & 88 & 90 & 75 & 81 & 94 & 285 & 440 \\
\hline 9 & 78 & 88 & 95 & 75 & 94 & 94 & 365 & 480 \\
\hline 10 & 73 & 90 & 90 & 88 & 81 & 81 & 310 & 680 \\
\hline 11 & 80 & 98 & 97 & 75 & 94 & 100 & 270 & 440 \\
\hline 12 & 70 & 93 & 95 & 58 & 92 & 100 & 210 & 333 \\
\hline 13 & 78 & 95 & 100 & 75 & 100 & 100 & 255 & 520 \\
\hline 14 & 93 & 80 & 88 & 94 & 100 & 100 & 210 & 160 \\
\hline 15 & 80 & 98 & 100 & 88 & 100 & 100 & 420 & 360 \\
\hline 16 & 88 & 99 & 99 & 81 & 100 & 100 & 390 & 520 \\
\hline Mean & 77 & 91 & 94 & 71 & 88 & 95 & 406 & 451 \\
\hline \multicolumn{9}{|l|}{ ISC } \\
\hline 1 & 60 & 88 & 96 & 56 & 81 & 94 & 390 & 560 \\
\hline 2 & 71 & 96 & 99 & 75 & 94 & 100 & 295 & 280 \\
\hline 3 & 72 & 83 & 97 & 58 & 92 & 92 & 525 & 720 \\
\hline 4 & 70 & 90 & 98 & 67 & 75 & 100 & 320 & 320 \\
\hline Mean & 68 & 89 & 98 & 64 & 86 & 97 & 383 & 470 \\
\hline \multicolumn{9}{|l|}{ RF1 } \\
\hline 1 & 62 & 90 & 93 & 58 & 67 & 92 & 690 & 320 \\
\hline 2 & 76 & 90 & 94 & 44 & 81 & 94 & 653 & 400 \\
\hline 3 & 78 & 90 & 92 & 67 & 75 & 100 & 675 & 360 \\
\hline 4 & 73 & 88 & 86 & 50 & 81 & 88 & 210 & 320 \\
\hline Mean & 72 & 90 & 91 & 55 & 76 & 94 & 557 & 350 \\
\hline \multicolumn{9}{|l|}{ RF2 } \\
\hline 1 & 73 & 86 & 91 & 38 & 88 & 94 & 615 & 1080 \\
\hline 2 & 74 & 86 & 91 & 63 & 75 & 75 & 360 & 680 \\
\hline 3 & 75 & 81 & 90 & 63 & 81 & 100 & 1000 & 800 \\
\hline 4 & 80 & 88 & 97 & 67 & 92 & 100 & 1000 & 1160 \\
\hline 5 & 84 & 91 & 91 & 63 & 88 & 94 & 670 & 480 \\
\hline Mean & 77 & 86 & 92 & 59 & 85 & 93 & 729 & 840 \\
\hline \multicolumn{9}{|l|}{ IBO1 } \\
\hline 1 & 74 & 90 & 96 & 44 & 88 & 100 & 670 & 480 \\
\hline 2 & 73 & 88 & 93 & 31 & 75 & 94 & 435 & 680 \\
\hline 3 & 79 & 80 & 95 & 56 & 75 & 94 & 390 & 560 \\
\hline 4 & 81 & 99 & 99 & 56 & 94 & 100 & 165 & 440 \\
\hline 5 & 76 & 91 & 93 & 50 & 69 & 94 & 180 & 360 \\
\hline Mean & 77 & 90 & 95 & 47 & 80 & 96 & 368 & 504 \\
\hline
\end{tabular}

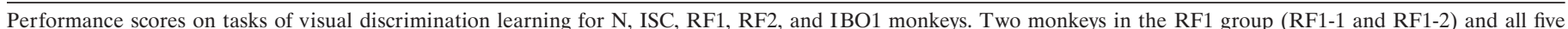

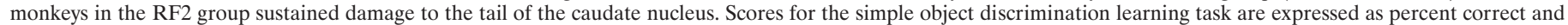

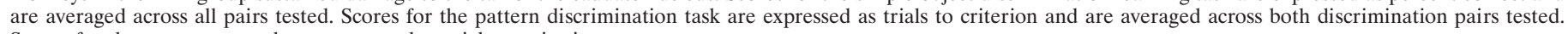
Scores for the concurrent task are expressed as trials to criterion. 

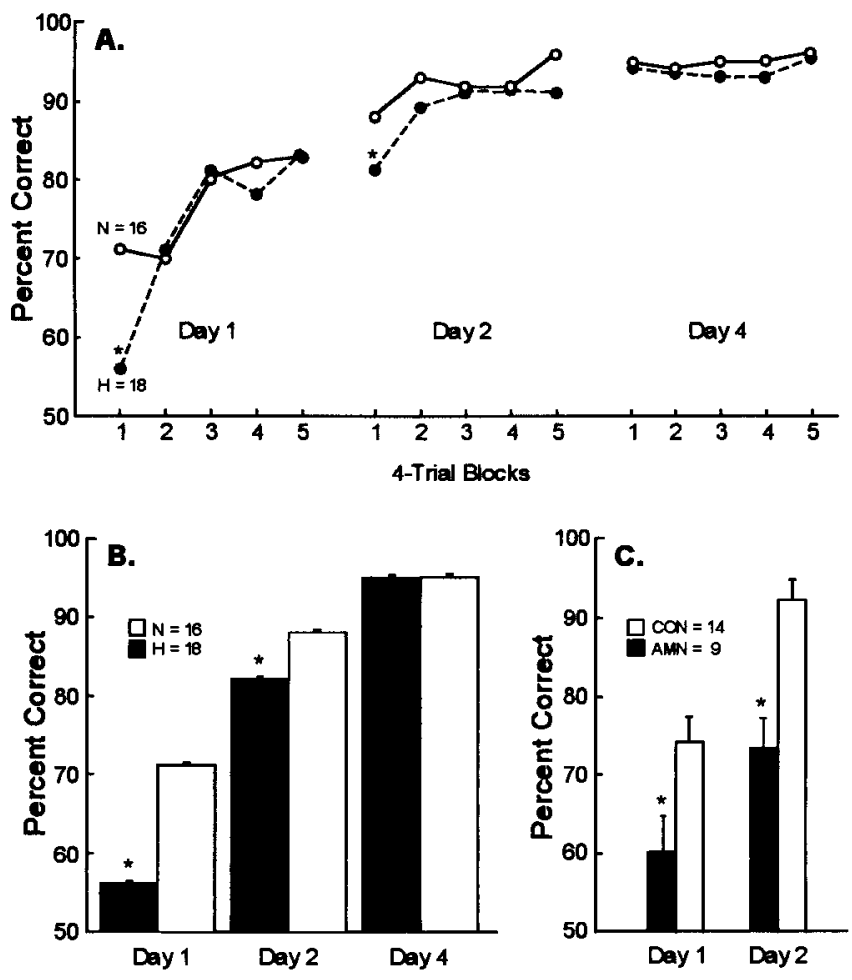

Figure 2. Performance on the simple object discrimination learning task. $A$, Learning curves (in blocks of 4 trials) for 18 monkeys with lesions of the hippocampal region ( $\mathrm{H}$, filled circles) and 16 normal monkeys ( $\mathrm{N}$, open circles) across the 3 test days. Performance scores have been averaged across four separate object discrimination tasks. Range of SEs: group N, 0.012-0.033; group H, 0.013-0.028. $B$, Mean percent correct scores for the first four trials of testing of each day for the $\mathrm{H}$ ( filled bars) and the $\mathrm{N}$ (open bars) groups. The 18 monkeys in the $\mathrm{H}$ group consist of four monkeys with ischemic lesions (ISC), nine monkeys with radio frequency lesions (RF1 and RF2), and five monkeys with ibotenate lesions (IBO1). C, Mean percent correct scores for the first four trials of testing of each day for nine amnesic patients (filled bars) and 14 controls (open bars) (from Squire et al., 1988). For the humans, the scores are the averages from three separate object discrimination tasks. For $B$ and $C$, brackets show SEM. ${ }^{*} p<0.05$.

evidence for an impairment in the first few trials of both days 1 and 2.

Performance on the first four trials of the object discrimination task was also analyzed by considering the four lesion groups separately (Table 2). On day 1 , the $\mathrm{RF} 1(p<0.05)$ and IBO1 $(p<0.01)$ groups were impaired relative to the $\mathrm{N}$ group, and the RF2 group was marginally impaired $(p<0.08)$. The ISC group also performed poorly (64\% correct), but the difference between this group and the $\mathrm{N}$ group (71\% correct) did not reach significance $(p>0.10)$. On day 2 , only the RF1 group was impaired $(p<0.05)$. None of the lesion groups was impaired on day $4(p>0.10)$.

To evaluate the possible contribution of damage to the caudate nucleus on object discrimination performance, we examined performance on the first four trials of each test day for the seven monkeys that sustained any damage to the caudate nucleus and for the remaining 11 operated monkeys without damage to the caudate nucleus. These two subgroups performed almost identically on the object discrimination task. On days 1,2 , and 4 , the seven monkeys with caudate damage obtained scores of 56,82 , and $93 \%$ correct, respectively. The corresponding scores for the 11 monkeys without caudate damage were 56, 82, and $96 \%$ correct. On day 1, both subgroups of monkeys were impaired relative to the $\mathrm{N}$ group (56 and 56 vs $71 \%$ correct; $p$ values $<$ $0.05)$. Across all three test days, the seven monkeys with caudate damage scored $77 \%$ correct, the 11 monkeys without caudate damage scored $78 \%$ correct, and the monkeys in the $\mathrm{N}$ group scored $85 \%$ correct. Both subgroups of operated monkeys were marginally impaired $(p=0.070)$.

A similar analysis of the first four trials was performed for the five monkeys who sustained damage to the hippocampal region, as well as inadvertent damage to the parahippocampal cortex, or to entorhinal or perirhinal cortex (RF1-3, IBO1-1, IBO1-3, IBO1-4, and IBO1-5). On day 1, both the five monkeys with cortical damage $(55 \%$ correct) and the remaining 13 monkeys without cortical damage ( $56 \%$ correct) were impaired $(\mathrm{N}, 71 \%$ correct; $p<0.05)$. The two subgroups performed better on the subsequent test days (day 2, 80 and 82\%, respectively; day 4, 98 and $94 \%$, respectively), although not significantly worse than the $\mathrm{N}$ group $(p>0.05)$. Across all 3 test days, the 13 monkeys without cortical lesions (77\% correct) were impaired (N, 85\% correct; $p<$ $0.05)$. The five monkeys with cortical lesions ( $77 \%$ correct) also performed more poorly than the $\mathrm{N}$ group, but this difference did not reach significance $(p>0.10)$.

In summary, the pattern of performance on the object discrimination task was similar for the monkeys with lesions limited to the hippocampal region and for the monkeys with lesions of the hippocampal region that included inadvertent damage to the tail of the caudate nucleus or to adjacent cortex. Thus, insofar as we could determine, there was no evidence that inadvertent damage to the tail of the caudate nucleus or cortical damage adjacent to the hippocampus contributed in a systematic way to object discrimination performance.

\section{Pattern discrimination}

Table 2 shows the performance scores of the N, ISC, RF1, RF2, and IBO1 groups on the pattern discrimination task. The number of trials required to learn the two pattern discrimination tasks were averaged for each monkey. Monkeys N5, RF2-3, and RF2-4 failed to reach criterion level of performance on either problem and testing was discontinued after 1000 trials. These animals were assigned a mean score of 1000 trials. Monkey N7 failed to reach a criterion level of performance on one of the problems, and testing was discontinued after 1000 trials. This animal was assigned a score of 1000 trials for that problem. Overall, the 18 monkeys with lesions of the hippocampal region (513 trials to criterion) were not different from the $\mathrm{N}$ group (406 trials to criterion; $p>0.10)$.

Examination of the performance of each group indicated that the ISC, RF1, and IBO1 groups all performed quite similarly to the $\mathrm{N}$ group. However, the monkeys in the RF2 group, all of which sustained significant bilateral damage to the tail of the caudate nucleus, differed from the $\mathrm{N}$ group (RF2, 729 trials to criterion; $\mathrm{N}, 406$ trials to criterion; $p<0.05$ ). The RF2 group also required significantly more trials to learn the pattern discrimination tasks than either the ISC group or the IBO1 group $(p<$ 0.05). Interestingly, two of the four monkeys in the RF1 group also sustained unilateral caudate damage, and the RF1 group also performed a little worse than the other groups without caudate damage (N, IBO1, and ISC). However, none of the pairwise comparisons was significant ( $p$ values $>0.10)$.

To evaluate further the contribution of damage to the caudate nucleus, we compared performance on the pattern discrimination task for all seven monkeys that sustained any damage to the 


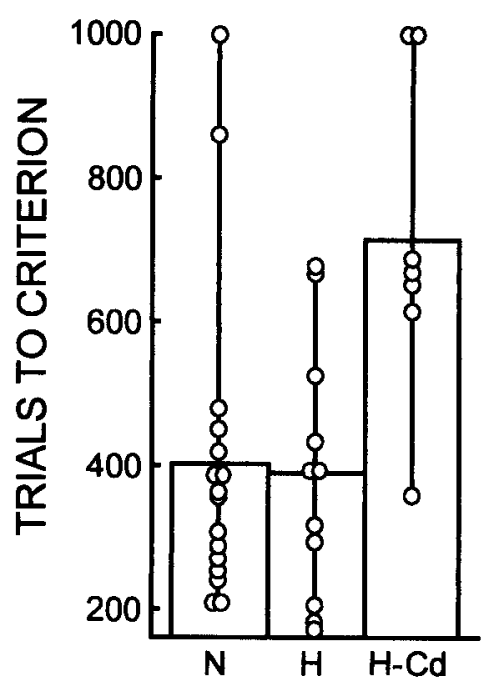

Figure 3. Performance on the pattern discrimination task for 16 normal control monkeys $(N), 11$ monkeys with lesions of the hippocampal region $(H)$, and seven monkeys with lesions of the hippocampal region that included substantial damage to the tail of the caudate nucleus (H-Cd). The 11 monkeys in the $\mathrm{H}$ group consist of four monkeys with ischemic lesions (ISC), two monkeys with radio frequency lesions (RF1-3 and RF1-4), and five monkeys with ibotenate lesions (IBO1). The seven monkeys in the $\mathrm{H}-\mathrm{Cd}$ group all had radio frequency lesions (RF1-1, RF1-2, and the 5 monkeys in the RF2 group). Performance scores for the two pattern discrimination problems are averaged together. Symbols show the performance of individual monkeys.

caudate nucleus (the five monkeys in the RF2 group and monkeys RF1-1 and RF1-2) with the performance of the remaining 11 operated monkeys without damage to the caudate nucleus (Fig. 3 ). The seven monkeys with caudate nucleus damage (713 trials to criterion) were significantly impaired, both with respect to the $\mathrm{N}$ group (406 trials) and to the 11 other monkeys without caudate damage ( 387 trials; $p$ values $<0.01$ ). Thus, damage to the caudate nucleus can account for impaired pattern discrimination learning performance in the monkeys with damage to the hippocampal region.

A similar analysis comparing the five monkeys who sustained damage to the parahippocampal cortex or to entorhinal or perirhinal cortex (RF1-3, IBO1-1, IBO1-3, IBO1-4, and IBO1-5) with the other 13 operated monkeys revealed no differences between the two subgroups (420 and 566 trials to criterion, respectively; $p>0.10$ ).

Finally, we asked whether the 11 monkeys with lesions limited to the hippocampal region who acquired the pattern discriminations normally might have been impaired during the early trials of learning as they were for simple object discrimination learning. We examined performance in five-trial blocks (30 trials each day) for the first $6 \mathrm{~d}$ of training (i.e., until some monkeys in each group reached criterion performance). The group with hippocampal lesions and the normal group averaged within $3.9 \%$ of each other at all 36 comparison points (all $p>0.10$, with the exception of trials $11-15$ on the first day of training, $p<0.05$ ). Thus, the two groups had very similar learning curves, and with the exception of one data point, the monkeys with lesions limited to the hippocampal region exhibited no signs of impairment early in training.

\section{Concurrent discrimination}

Table 2 shows the performance scores of the N, ISC, RF1, RF2, and IBO1 groups on the concurrent discrimination task. Overall,

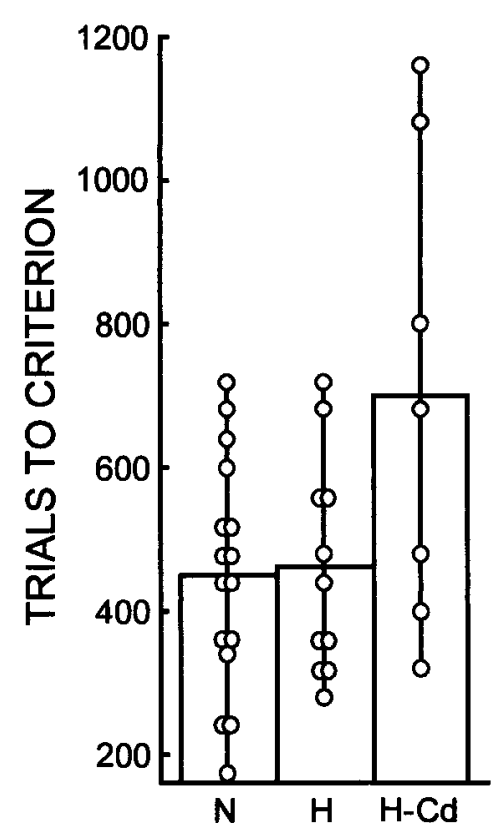

Figure 4. Performance on the concurrent discrimination task for 16 normal control monkeys $(\mathrm{N}), 11$ monkeys with lesions of the hippocampal region $(\mathrm{H})$, and seven monkeys with lesions of the hippocampal region that included substantial damage to the tail of the caudate nucleus $(\mathrm{H}-\mathrm{Cd})$. See Figure 3 for the composition of the two lesion groups. Symbols show the performance of individual monkeys.

the 18 monkeys with lesions of the hippocampal region (556 trials to criterion) did not differ from the $\mathrm{N}$ group (451 trials to criterion; $p>0.10$ ). Learning occurred gradually across sessions such that performance improved incrementally for each of the eight object pairs. Performance of the 16 normal monkeys averaged 57, 59, 65, and $72 \%$ correct across the first four test sessions (averaged across all eight object pairs).

Examination of the performance of each group indicated that the ISC, RF1, and IBO1 groups all performed similarly to the N group. However, the monkeys in the RF2 group, all of which sustained caudate damage, performed more poorly than the $\mathrm{N}$ group (RF2, 840 trials to criterion; N, 541 trials to criterion; $p<$ $0.001)$. The RF2 group also required significantly more trials to learn the concurrent discrimination task than either the RF1 group or the IBO1 group $(p<0.05)$ and marginally more trials than the ISC group $(p<0.07)$.

To evaluate further the contribution of damage to the caudate nucleus, we compared performance on the concurrent discrimination task for all seven monkeys that sustained any damage to the caudate nucleus with the performance of the remaining 11 operated monkeys without damage to the caudate nucleus (Fig. 4). The seven monkeys with caudate nucleus damage (703 trials to criterion performance) were significantly impaired with respect to both the $\mathrm{N}$ group (451 trials) and the 11 other monkeys without caudate damage (462 trials; $p$ values $<0.05$ ). Thus, as in the case of pattern discrimination learning, damage to the caudate nucleus appears to account for the impaired concurrent discrimination learning performance in the monkeys with damage to the hippocampal region.

A similar analysis comparing the five monkeys who sustained damage to the parahippocampal cortex (or to entorhinal or perirhinal cortex) with the remaining 13 operated monkeys with- 
out cortical damage revealed no differences between the two subgroups (368 vs 600 trials to criterion; $p>0.10$ ).

Finally, we asked whether the 11 monkeys with lesions limited to the hippocampal region, who acquired the concurrent discrimination task normally, might have been impaired during the early trials of learning. We examined performance in five-trial blocks (40 trials per day) for the first $5 \mathrm{~d}$ of training (i.e., until some monkeys in each group reached criterion performance). The group with hippocampal lesions and the normal group averaged within $3.2 \%$ of each other across the 40 comparison points (all $p$ values $>0.10)$. Thus, the groups had very similar learning curves, and the monkeys with lesions limited to the hippocampal region exhibited no sign of impairment early in training.

\section{Lateral geniculate nucleus}

Of the seven monkeys with damage to the caudate nucleus, five also sustained damage to the LGN (the five monkeys in the RF2 group). We considered the possibility that a visual impairment could account for the behavioral deficit of these monkeys on the pattern discrimination and the concurrent discrimination tasks. It is unlikely, however, that this possibility can explain the pattern of findings in the seven monkeys. First, the damage to the LGN was only partial (19-45\%; see Neurohistological findings). Second, the stimuli used in the object discrimination task and the concurrent discrimination task are similar, yet performance was intact on the object discrimination task. Third, behavioral data for the RF2 group (which had hippocampal damage plus damage to the LGN) were reported in our recent study of object recognition (Zola et al., 2000). In that study, the RF2 group performed entirely normally at the $1 \mathrm{sec}$ delay on the visual pairedcomparison task, which involved black-and-white line drawings. They were impaired at longer delays. Finally, the RF2 group was normal at acquiring the delayed nonmatching to sample task with trial-unique objects (at an 8 sec delay) but was impaired at longer delays. These findings rule out the possibility that the behavioral deficit on the pattern discrimination and the concurrent discrimination tasks in the monkeys with damage to the LGN was attributable to a visual impairment.

\section{DISCUSSION}

There were two main findings. First, the integrity of the hippocampal region is required for normal learning and retention of the simple, two-choice object discrimination task. Monkeys with ischemic lesions, radio frequency lesions, or ibotenate lesions of the hippocampal region were impaired at object discrimination learning, especially during the first several trials of the first $2 \mathrm{~d}$ of testing (Fig. $2 A, B$ ). Second, pattern discrimination learning and concurrent discrimination learning are dependent on the integrity of the caudate nucleus. Monkeys with hippocampal lesions who additionally sustained damage to the tail of the caudate nucleus were impaired on both of these discrimination tasks. However, monkeys with hippocampal lesions who did not sustain caudate damage performed both of these tasks normally.

Studies of simple discrimination learning in human amnesic patients (Squire et al., 1988) have also shown that the first few trials of each testing day are the most sensitive to memory impairment (Fig. 2C). The reason why the first few trials of each test day are especially sensitive to amnesia is straightforward. Amnesic patients are forgetful and have two kinds of difficulty with object discrimination learning. First, during the early trials of each test day, they have difficulty remembering the feedback they receive concerning which object is the correct one. Second, they forget what they have learned from day to day. Because the patients typically have some residual memory ability that allows them to benefit from repetition, the later training trials of each test day are less sensitive to amnesia than the early trials. In addition, the patients can rehearse the correct answer once they have determined what it is.

Mishkin and his colleagues first proposed that the basal ganglia, including the caudate nucleus and its corticostriatal inputs, subserve the formation of habits (Mishkin et al., 1984). By this view, the caudate nucleus is part of a corticostriatal system that associates sensory inputs processed in the cortex with responses generated by the extrapyramidal system, yielding stimulus-response associations that constitute habits. What is stored is the changing probability that a stimulus will elicit a particular response. In the case of visual habits, the relevant connectivity is proposed to involve higher visual areas in inferior temporal cortex (e.g., area TE) and projections from inferotemporal cortex to the tail of the caudate nucleus (Phillips et al., 1988).

Several findings have suggested that both the pattern discrimination task and the concurrent discrimination task can be accomplished as tasks of habit learning (Mishkin and Petri, 1984; Zola-Morgan and Squire, 1984; Phillips et al., 1988; Zola-Morgan et al., 1994; Buffalo et al., 1998). First, a factor analysis of four tasks that have been used to measure memory in the monkey indicated that the pattern discrimination task and the concurrent discrimination task are distinct from other tasks that depend on the integrity of the medial temporal lobe (e.g., the delayed nonmatching to sample task) (Zola-Morgan et al., 1994). Unlike tasks that depend on the medial temporal lobe, pattern discrimination and concurrent discrimination learning are accomplished gradually, day by day, over hundreds of trials, and performance improves incrementally as training continues (Moss et al., 1981; Squire and Zola-Morgan, 1983; Zola-Morgan and Squire, 1984). Second, pattern discrimination learning in monkeys was impaired by damage to the tail of the caudate nucleus (Divac et al., 1967). Third, in a preliminary report, monkeys with damage to the tail of the caudate nucleus were impaired in concurrent discrimination learning (Wang et al., 1990). Finally, lesions of area TE, which projects to the tail of the caudate nucleus, impair performance in both pattern discrimination learning and concurrent discrimination learning (Gross, 1973; Dean, 1976; Phillips et al., 1988; Buffalo et al., 1998, 1999).

The present findings provide additional support for this idea that the pattern discrimination and concurrent discrimination tasks can be accomplished as tasks of habit learning. Both tasks were acquired normally despite damage to the hippocampal region, a component of the medial temporal lobe memory system important for declarative memory. In contrast, damage to the tail of the caudate nucleus impaired performance on both tasks.

The nature of the simple object discrimination task contrasts sharply with the nature of pattern discrimination and concurrent discrimination learning. When only a single pair of objects needs to be learned and the objects are easily discriminable, as in the simple object discrimination task, learning which stimulus is correct becomes the major work of the task. Although it has sometimes been suggested that simple object discrimination tasks are insensitive to medial temporal lobe lesions (Gaffan, 1994; Doré et al., 1998), an earlier review of the data from 46 twochoice discrimination tasks found that the tasks learned quickly by normal animals were more sensitive to medial temporal lobe lesions than the tasks learned gradually (Squire and ZolaMorgan, 1983). More recent studies of simple two-choice visual 
discrimination tasks are also consistent with this idea (Murray et al., 1998; Baxter et al., 1999).

The differences between simple object discrimination learning, concurrent discrimination learning, and pattern discrimination learning are particularly apparent in the dissociations that can be demonstrated between the effects of medial temporal lobe lesions and lesions of adjacent inferotemporal cortical area TE (Buffalo et al., 1999). For example, perirhinal cortex lesions impair performance on the simple object discrimination task but do not impair performance on pattern discrimination learning or on the eight-pair concurrent discrimination task. In contrast, lesions of area TE impair performance on both the concurrent discrimination and pattern discrimination tasks but do not impair performance on the simple object discrimination task (Buffalo et al., 1999).

In summary, the current findings provide evidence for a distinction in the monkey between the functions of the hippocampal region and the caudate nucleus. The hippocampus is a component of the medial temporal lobe memory system important for rapid learning (for a recent perspective, see Wise and Murray, 1999). Thus, the hippocampus is important for single-trial tasks of recognition memory (Zola et al., 2000), as well as for rapidly learned simple object discrimination tasks (the present study). In contrast, the caudate nucleus is part of a corticostriatal system that subserves the gradual learning of habits and stimulus-reward associations (for review, see Graybiel, 1995). Recent work points to a similar distinction in the rodent literature (Packard et al., 1989; Packard and McGaugh, 1992), as well as in the human neuropsychological literature, between fast declarative learning and slower habit learning (Phillips and Carr, 1987; Salmon and Butters, 1995; Knowlton et al., 1996).

\section{REFERENCES}

Alvarez P, Zola-Morgan S, Squire LR (1995) Damage limited to the hippocampal region produces long-lasting memory impairment in monkeys. J Neurosci 15:3796-3807.

Baxter MG, Hadfield WS, Murray EA (1999) Rhinal cortex lesions produce mild deficits in visual discrimination learning for an auditory secondary reinforcer in rhesus monkeys. Behav Neurosci 113:243-252.

Buffalo EA, Stefanacci L, Squire LR, Zola SM (1998) A reexamination of the concurrent discrimination learning task: the importance of inferotemporal cortex, area TE. Behav Neurosci 112:3-14.

Buffalo EA, Ramus SJ, Clark RE, Teng E, Squire LR, Zola SM (1999) Dissociation between the effects of damage to perirhinal cortex and Area TE. Learn Mem 6:572-599.

Dean P (1976) Effects of inferotemporal lesions on the behavior of monkeys. Psychol Bull 83:41-71.

Divac I, Rosvold HE, Szwarcbart MK (1967) Behavioral effects of selective ablation of the caudate nucleus. J Comp Physiol Psychol 63:184-190.

Doré FY, Thornton JA, White NM, Murray EA (1998) Selective hippocampal lesions yield nonspatial memory impairment in rhesus monkeys. Hippocampus 8:323-329.

Gaffan D (1994) Dissociated effects of perirhinal cortex ablation, fornix transection and amygdalectomy: evidence for multiple memory systems in the primate temporal lobe. Exp Brain Res 99:411-422.

Gellerman LW (1933) Chance orders of alternating stimuli in visual discrimination experiments. J Genet Psychol 42:207-208.

Graybiel AM (1995) Building action repertoires: memory and learning functions of the basal ganglia. Curr Opin Neurobiol 5:733-741.

Gross CG (1973) Visual functions of inferotemporal cortex. In: Handbook of sensory physiology (Autrum H, Jung R, Lowenstein WR, McKay DM, Teuber HL, eds), pp 451-482. Berlin: Springer.

Iversen SD (1976) Do hippocampal lesions produce amnesia in animals? Int Rev Neurobiol 19:1-49.

Jones B, Mishkin M (1972) Limbic lesions and the problem of stimulusreinforcement associations. Exp Neurol 36:362-377.
Knowlton BJ, Mangels JA, Squire LR (1996) A neostriatal habit learning system in humans. Science 273:1399-1402.

Mahut H, Moss M, Zola-Morgan S (1981) Retention deficits after combined amygdalo-hippocampal and selective hippocampal resections in the monkey. Neuropsychologia 19:201-225.

Mishkin M, Murray EA (1994) Stimulus recognition. Curr Opin Neurobiol 4:200-206.

Mishkin M, Petri HL (1984) Memories and habits: some implications for the analysis of learning and retention. In: Neuropsychology of memory (Squire LR, Butters N, eds), pp 287-296. New York: Guilford.

Mishkin M, Malamut B, Bachevalier J (1984) Memories and habits: two neural systems. In: Neurobiology of human learning and memory (Lynch G, McGaugh JL, Weinberger NM, eds), pp 65-77. New York: Guilford.

Mishkin M, Suzuki WA, Gadian DG, Vargha-Khadem F (1999) Hierarchical organization of cognitive memory. Phil Trans R Soc Lond B Biol Sci 352:1461-1467.

Moss M, Mahut H, Zola-Morgan S (1981) Concurrent discrimination learning of monkeys after hippocampal, entorhinal, or fornix lesions. J Neurosci 1:227-240.

Murray EA, Baxter MG, Gaffan D (1998) Monkeys with rhinal cortex damage or neurotoxic hippocampal lesions are impaired on spatial scene learning and object reversals. Behav Neurosci 112:1291-1303.

Orbach J, Milner B, Rasmussen T (1960) Learning and retention in monkeys after amygdala-hippocampus resection. Arch Neurol 3:230-251.

Packard MG, McGaugh JL (1992) Double dissociation of fornix and caudate nucleus lesions on acquisition of two water maze tasks: further evidence for multiple memory systems. Behav Neurosci 106:439-446.

Packard MG, Hirsh R, White NM (1989) Differential effects of fornix and caudate nucleus lesions on two radial maze tasks: evidence for multiple memory systems. J Neurosci 9:1465-1472.

Phillips AG, Carr GD (1987) Cognition and the basal ganglia: a possible substrate for procedural knowledge. Can J Neurol Sci 14:381-385.

Phillips RR, Malamut BL, Bachevalier J, Mishkin M (1988) Dissociation of the effects of inferior temporal and limbic lesions on object discrimination learning with 24-h intertrial intervals. Behav Brain Res 27:99-107.

Rempel-Clower N, Zola SM, Squire LR (1996) Three cases of enduring memory impairment following bilateral damage limited to the hippocampal formation. J Neurosci 16:5233-5255.

Salmon DP, Butters N (1995) Neurobiology of skill and habit learning. Curr Opin Neurobiol 5:184-190.

Squire LR, Zola-Morgan S (1983) The neurology of memory: the case for correspondence between the findings for human and nonhuman primate. In: The physiological basis of memory (Deutsch JA, ed), pp 199-268. New York: Academic.

Squire LR, Zola-Morgan S (1991) The medial temporal lobe memory system. Science 253:1380-1386.

Squire LR, Zola-Morgan S, Chen K (1988) Human amnesia and animal models of amnesia: performance of amnesic patients on tests designed for the monkey. Behav Neurosci 11:210-221.

Suzuki WA, Zola-Morgan S, Squire LR, Amaral DG (1993) Lesions of the perirhinal and parahippocampal cortices in the monkey produce long-lasting memory impairment in the visual and tactual modalities. J Neurosci 13:2430-2451.

Szabo J, Cowan WM (1984) A stereotaxic atlas of the brain of the cynomolgus monkey (Macaca fascicularis). J Comp Neurol 222:265-300.

Wang J, Aigner T, Mishkin M (1990) Effects of neostriatal lesions on visual habit formation in rhesus monkeys. Soc Neurosci Abstr 16:617.

Wise SP, Murray EA (1999) Role of the hippocampal system in conditional motor learning: Mapping antecedents to action. Hippocampus 9:101-117.

Zola S, Squire LR (2000) Memory and brain systems. In: Oxford handbook of memory (Tulving E, Craik F, eds). Oxford: Oxford UP.

Zola SM, Squire LR, Teng E, Stefanacci L, Buffalo E, Clark RE (2000) Impaired recognition memory in monkeys after damage limited to the hippocampal region. J Neurosci 20:451-463.

Zola-Morgan S, Squire LR (1984) Preserved learning in monkeys with medial temporal lesions: sparing of motor and cognitive skills. J Neurosci 4:1072-1085.

Zola-Morgan S, Squire LR (1985) Medial temporal lesions in monkeys impair memory on a variety of tasks sensitive to human amnesia. Behav Neurosci 99:22-34. 
Zola-Morgan S, Squire LR, Amaral DG (1986) Human amnesia and the medial temporal region: enduring memory impairment following a bilateral lesion limited to field CA1 of the hippocampus. J Neurosci 6:2950-2967.

Zola-Morgan S, Squire LR, Amaral DG (1989a) Lesions of the hippocampal formation but not lesions of the fornix or the mammillary nuclei produce long-lasting memory impairment in monkeys. J Neurosci 9:898-913.

Zola-Morgan S, Squire LR, Amaral DG (1989b) Lesions of the amygdala that spare adjacent cortical regions do not impair memory or exacerbate the impairment following lesions of the hippocampal formation. J Neurosci 9:1922-1936.

Zola-Morgan S, Squire LR, Amaral DG, Suzuki WA (1989c) Lesions of perirhinal and parahippocampal cortex that spare the amygdala and hippocampal formation produce severe memory impairment. J Neurosci 9:4355-4370.

Zola-Morgan S, Squire LR, Rempel NL, Clower RP, Amaral DG (1992) Enduring memory impairment in monkeys after ischemic damage to the hippocampus. J Neurosci 9:4355-4370.

Zola-Morgan S, Squire LR, Clower RP, Rempel NL (1993) Damage to the perirhinal cortex exacerbates memory impairment following lesions to the hippocampal formation. J Neurosci 13:251-265.

Zola-Morgan S, Squire LR, Ramus SJ (1994) Severity of memory impairment in monkeys as a function of locus and extent of damage within the medial temporal lobe memory system. Hippocampus $4: 483-495$ 